\title{
The Estimation of the Spot Volatility for Diffusion Process
}

\author{
Weiwei Xu ${ }^{1}$, Xin Yang ${ }^{2 *}$, Shanchao Yang ${ }^{1}$ \\ ${ }^{1}$ School of Mathematics and Statistics, Guangxi Normal University, Guilin, China \\ ${ }^{2}$ School of Mathmatical Sciences, Guilin University of Aerospace Technology, Guilin, China \\ Email: *xinyang_emily@sina.cn
}

How to cite this paper: $\mathrm{Xu}, \mathrm{W} . \mathrm{W} .$, Yang, $X$. and Yang, S.C. (2021) The Estimation of the Spot Volatility for Diffusion Process. Open Journal of Statistics, 11, 303-318. https://doi.org/10.4236/ojs.2021.112017

Received: March 4, 2021

Accepted: April 18, 2021

Published: April 21, 2021

Copyright $\odot 2021$ by author(s) and Scientific Research Publishing Inc. This work is licensed under the Creative Commons Attribution International License (CC BY 4.0).

http://creativecommons.org/licenses/by/4.0/

\begin{abstract}
In this paper, we propose a Gasser-Müller type spot volatility estimator (abbreviated as GM type estimator) for diffusion process, which is weighted by integrals, it is different from the kernel spot volatility estimator discussed by Kristensen (2010). Under more general conditions, the asymptotic unbiasedness and the asymptotic normality of the GM type estimator are derived. The simulation results show that the GM type spot volatility estimator has good estimation effect, and its mean square error tends to be less than that of the kernel spot volatility estimator discussed by Kristensen (2010), so it provides a selection method for estimating the spot volatility in high frequency data environment.
\end{abstract}

\section{Keywords}

Diffusion Process, Spot Volatility, GM Type Estimation, Asymptotic Property

\section{Introduction}

Volatility is the main component of describing the price movement towards financial market, so we need to use the observed actual data to estimate volatility. However, the traditional low-frequency data cannot meet the needs of high-frequency traders in today's market, and the common models in the traditional low-frequency field are no longer applicable to the high-frequency field. In order to avoid errors caused by setting any model, more and more scholars consider using non-parametric methods to estimate volatility in high-frequency data.

Consider the diffusion process $\left\{X_{t}\right\}=\left\{X_{t}: t \geq 0\right\}$, which satisfies that

$$
\mathrm{d} X_{t}=\mu_{t} \mathrm{~d} t+\sigma_{t} \mathrm{~d} W_{t},
$$


where $\left\{W_{t}\right\}$ is a standard Brownian motion, while $\left\{\mu_{t}\right\}$ and $\left\{\sigma_{t}\right\}$ are adapted stochastic processes. The process $\left\{\sigma_{t}\right\}$ is usually denoted the spot volatility process, while $\left\{\mu_{t}\right\}$ is the drift process. In the diffusion process, the volatility is an important measure to reflect the volatility of financial products' logarithmic price.

Under the condition that asset prices obey the diffusion process, the quadratic variation of the price process converges in probability to the integrated volatility. So quadratic variation, also known as realized volatility, is a consistent estimator of integrated volatility, and has attracted more and more attention. For example, Barndorff-Nielsen and Shephard [1] (2002) prove the central limit theorem of realized volatility. Christensen and Podolskij [2] (2005) give another estimator of integral volatility, range-based realized volatility, and prove the consistency and central limit theorem of this estimator under diffusion model. A nonparametric estimator is proposed by Li Guo and Bao [3] (2013) for the class of integrated self-weighted cross volatilities. Under assuming that processes $\mathrm{X}$ and $\mathrm{Y}$ are sampled with microstructure noise and in an asynchronous way, the asymptotic properties, which include consistency and asymptotic normality, are obtained. There is still a lot of further research on the integrated volatility estimator, see also, Zhang, Mykland and At-Sahalia [4] (2005), Hansen, Large and Lunde [5] (2008), Li, et al. [6] (2013), Jing and Liu and Kong [7] (2015) and Li, Xie and Zheng [8] (2016).

Merton [9] (1976) first proposed the jump-diffusion model. Later, Duffie, Pan and Singleton [10] (2000), Pan [11] (2002), Eraker, Johannes and Polson [12] (2003) found that asset price process contains improper mutation behavior, so jump part should be added in diffusion model. Previous studies have shown that integrated volatility is generally estimated by realized multiple power variation and realized threshold volatility when asset prices obey jump-diffusion model. Barndorff-Nielsen and Shephard [13] [14] $(2004,2006)$ proposed realized power variation and realized bipower variation, and proved that realized bipower variation is a robust consistent estimator of integrated volatility with jump process. By using the properties of Brown's motion path, when the price obeys the jump-diffusion model with finite jump, another estimator of integrated volatility, the realized threshold volatility, is proposed, and the central limit theorem is obtain by Mancini [15] [16] $(2004,2009)$. Christensen and Podolskij [17] (2012) put forward the realized multiple power variation of based-range. They also show the robustness of the estimator to jump behavior in probability limit and asymptotic distribution. The further research can be seen in Todorov [18] [19] $(2009,2010)$, At-Sahalia and Jacod [20] [21] $(2009,2011)$, Tauchen and Zhou [22] (2011).

The integrated volatility characterizes the total volatility over a certain period of time for the past, but investors pay more attention to the spot volatility at a certain time, so we need to use high-frequency data to estimate the spot volatility. As the price process obeys the diffusion process, the rolling sample estimator for spot volatility was first proposed by Foster and Nelson [23] (1996). This rolling 
sample method is extended by Andreou and Ghysels [24] (2002) to construct the rolling sample variance to estimate the quadratic variation of asset returns, and a rolling sample estimator with many similar characteristics to the instantaneous volatility estimator is proposed. They further discussed the asymptotically effective bandwidth and weighted form of the quadratic variation estimator, and established the relationship between the instantaneous volatility estimator and the integrated volatility estimator.

Moreover, Fan and Wang [25] (2008), Shigeyoshi and Simona [26] (2011), Zu and Boswijk [27] (2014), Yu, et al. [28] (2014), Mancini, Mattiussi and Renò [29] (2015), Curato, Mancino and Recchioni [30] (2018) and Liu, et al. [31] (2018) et al. have done a lot of research on instantaneous volatility estimation. Fan and Wang [25] (2008) employs a bivariate diffusion to model the price and volatility of an asset and investigates kernel type estimators of spot volatility based on high-frequency return data. And they establish both pointwise and global asymptotic distributions for the estimators. Zu and Peter [27] (2014) constructs a spot volatility estimate for high frequency financial data with market microstructure noise. At the same time, the consistency of the estimator is proved and the asymptotic distribution of the estimator is derived. Curato, Mancino and Recchioni [30] (2018) defines a new nonparametric estimation of spot volatility based on the relationship between Laplace transformation of price process and fluctuation process of Brownian semi-martingale model, and proves the consistency and asymptotic normality of the estimation. The simulation results confirm the theoretical results of the proposed estimator in the presence of noise effects on the microstructure.

According to the idea of Nadaraya-Watson kernel regression estimation, Kristensen [32] (2010) proposes the NW type kernel estimation of spot volatility $\sigma_{t}$

$$
\hat{\sigma}_{\tau}^{2}=\sum_{i=1}^{n} K_{h}\left(t_{i}-\tau\right)\left(\Delta X_{t_{i}}\right)^{2}, \tau \in[0, T]
$$

where $T$ is the time interval, $t_{i}=i \delta, i=0,1, \cdots, n, \delta=T / n, \Delta X_{t_{i}}=X_{t_{i}}-X_{t_{i-1}}$, $K_{h}(s-\tau)=K\left(\frac{s-\tau}{h}\right) / h, K: R \mapsto R$ is a kernel, $h>0$ is the bandwidth. And the asymptotic property of the kernel estimator is proved in Kristensen [32] (2010).

In non-parameter kernel regression estimation, there are two popular kernel methods, Nadaraya-Watson estimator $\hat{\mu}_{n, N W}(t)$ and Gasser-Müller estimator $\hat{\mu}_{n, G M}(t)$. Gasser and Müller [33] (1979) gave

$$
E\left(\hat{\mu}_{n, N W}(t)\right)=h^{-1} \int_{0}^{1} K\left(\frac{t-s}{h}\right) \mu(s) \mathrm{d} s+O\left(n^{-\gamma_{\mu}}+(n h)^{-\gamma_{K}}+n^{-\left(\delta_{0}-1\right)}\right)
$$

and

$$
E\left(\hat{\mu}_{n, G M}(t)\right)=h^{-1} \int_{0}^{1} K\left(\frac{t-s}{h}\right) \mu(s) \mathrm{d} s+O\left(n^{-\gamma_{\mu}}\right),
$$

where $\gamma_{\mu}>0, \gamma_{K}>0$ and $\delta_{0}>1$. The two equations imply that Gasser-Müller 
estimator $\hat{\mu}_{n, G M}(t)$ has a better remainder term for the bias than NadarayaWatson estimator $\hat{\mu}_{n, N W}(t)$.

In view of this advantage, this paper will study the Gasser-Müller integrated type spot volatility estimator (or the GM type spot volatility estimator)

$$
\hat{\sigma}_{\tau, G M}^{2}=\frac{1}{\delta} \sum_{i=1}^{n}\left(\Delta X_{t_{i}}\right)^{2} \int_{t_{i-1}}^{t_{i}} K_{h}(s-\tau) \mathrm{d} s, \tau \in[0, T] .
$$

We will demonstrate the asymptotic property of the GM type instantaneous volatility estimator under more general conditions, including asymptotic unbias and asymptotic normality.

The remainder of the paper is organized as follows. In Section 2, asymptotic property of the GM type instantaneous volatility estimator are proved. Simulation study is put in Section 3. Finally, our conclusion is given in Section 4.

\section{Asymptotic Property of Estimation}

Throughout the paper, we will use the following basic assumptions.

1) Let $\delta=T / n, t_{i}=i \delta, i=0,1, \cdots, n$.

2) (a) The processes $\left\{\mu_{t}\right\}$ and $\left\{\sigma_{t}\right\}$ are jointly independent of $\left\{W_{t}\right\}$, absolutely integral and locally bounded; (b) There exist constants $\lambda>0$ and $C>0$ such that

$$
E\left|\sigma_{t}-\sigma_{t}\right| \leq C|t-s|^{\lambda}
$$

(c) Let $f(t)=\sigma_{t}^{2}$. Assume that $f(t)$ is $m$ times differentiable where $m \geq 0$, and there exist constants $\alpha>0$ and $C>0$ such that

$$
E\left|f^{(m)}(t)-f^{(m)}(\tau)\right| \leq C|t-\tau|^{\alpha} .
$$

3) (a) The kernel $K(u)$ is bounded in $R^{1}$ and satisfies that $\int_{-\infty}^{+\infty} K(u) \mathrm{d} u=1$, $\int_{-\infty}^{+\infty}|K(u)| \mathrm{d} u<\infty, \int_{-\infty}^{+\infty} u^{j} K(u) \mathrm{d} u=0$ for $j=1,2, \cdots, m$, and $\int_{-\infty}^{-\infty}|u|^{r}|K(u)| \mathrm{d} u<\infty$ for some $r \geq m+\alpha$ and $r \geq \lambda$; (b) $K(u)$ satisfies the $\beta$ th order Lipschitz condition where $\beta>0$.

4) As $n \rightarrow \infty, h_{n} \rightarrow 0$ and $n h_{n}^{1+1 / \beta} \rightarrow \infty$.

Notice that when $f(t)$ is not differentiable, $m=0$ in (c) of (2). In this case, the conditions (2.1) and (2.2) are identical.

Bandi and Nguyen [34] (2003) and Bandi and Phillips [35] (2003) assumed that $\sigma_{t}$ and $\mu_{t}$ are at least twice continuously differentiable and satisfy Lipschitz condition. Kristensen [32] (2010) relaxed the restriction of differentiability and allowed the order of differentiability $m \geq 0$. Our assumption (2) is to refer to the assumption A.2 - A.4 in Kristensen [32] (2010).

In this section, we first establish the asymptotic unbias of the kernel estimator (1.5), and then study its asymptotic normality.

Theorem 2.1. Assume that the conditions (1) -(4) hold. Then

$$
E\left(\hat{\sigma}_{\tau, G M}^{2} \mid\left\{\sigma_{t}\right\}\right)-\sigma_{\tau}^{2}=O_{p}\left(n^{-1}+\frac{1}{n^{\beta} h_{n}^{1+\beta}}+h_{n}^{m+\alpha}\right),
$$

furthermore 


$$
E\left(\hat{\sigma}_{\tau, G M}^{2}-\sigma_{\tau}^{2}\right)=O\left(n^{-1}+\frac{1}{n^{\beta} h_{n}^{1+\beta}}+h_{n}^{m+\alpha}\right) .
$$

Proof We introduce some denotations. Let $\Delta \tilde{X}_{t_{i}}=\int_{t_{i-1}}^{t_{i}} \sigma_{s} \mathrm{~d} W_{s}$ and

$$
\tilde{\sigma}_{\tau, G M}^{2}=\frac{1}{\delta} \sum_{i=1}^{n}\left(\Delta \tilde{X}_{t_{i}}\right)^{2} \int_{t_{i-1}}^{t_{i}} K_{h}(s-\tau) \mathrm{d} s .
$$

Obviously,

$$
\begin{aligned}
\hat{\sigma}_{\tau, G M}^{2}-\tilde{\hat{\sigma}}_{\tau, G M}^{2}= & \frac{1}{\delta} \sum_{i=1}^{n}\left(\int_{t_{i-1}}^{t_{i}} \mu_{s} \mathrm{~d} s+\int_{t_{i-1}}^{t_{i}} \sigma_{s} \mathrm{~d} W_{s}\right)^{2} \int_{t_{i-1}}^{t_{i}} K_{h}(s-\tau) \mathrm{d} s \\
& -\frac{1}{\delta} \sum_{i=1}^{n}\left(\int_{t_{i-1}}^{t_{i}} \sigma_{s} \mathrm{~d} W_{s}\right)^{2} \int_{t_{i-1}}^{t_{i}} K_{h}(s-\tau) \mathrm{d} s \\
= & \frac{1}{\delta} \sum_{i=1}^{n}\left(\int_{t_{i-1}}^{t_{i}} \mu_{s} \mathrm{~d} s\right)^{2} \int_{t_{i-1}}^{t_{i}} K_{h}(s-\tau) \mathrm{d} s \\
& +\frac{2}{\delta} \sum_{i=1}^{n} \int_{t_{i-1}}^{t_{i}} \mu_{s} \mathrm{~d} s \int_{t_{i-1}}^{t_{i}} \sigma_{s} \mathrm{~d} W_{s} \int_{t_{i-1}}^{t_{i}} K_{h}(s-\tau) \mathrm{d} s .
\end{aligned}
$$

Due to boundedness of $\mu_{t}$, it follows that

$$
\begin{aligned}
\frac{1}{\delta}\left|\sum_{i=1}^{n}\left(\int_{t_{i-1}}^{t_{i}} \mu_{s} \mathrm{~d} s\right)^{2} \int_{t_{i-1}}^{t_{i}} K_{h}(s-\tau) \mathrm{d} s\right| & \leq C \delta \sum_{i=1}^{n} \int_{t_{i-1}}^{t_{i}}\left|K_{h}(s-\tau)\right| \mathrm{d} s \\
& =C \delta \int_{0}^{T}\left|K_{h}(s-\tau)\right| \mathrm{d} s \\
& \leq C \delta \int_{-\infty}^{\infty}|K(u)| \mathrm{d} u \\
& \leq C n^{-1} .
\end{aligned}
$$

Note that

$$
\int_{t_{i-1}}^{t_{i}} \sigma_{s} \mathrm{~d} W_{s} \stackrel{\operatorname{law}}{=}\left(\int_{t_{i-1}}^{t_{i}} \sigma_{s}^{2} \mathrm{~d} s\right)^{1 / 2} U_{i}
$$

where $U_{1}, U_{2}, \cdots, U_{n}$ are independently and identically standard normal $N(0,1)$ and independent of the process $\sigma$. Let

$$
A_{n}=\frac{2}{\delta} \sum_{i=1}^{n} \int_{t_{i-1}}^{t_{i}} K_{h}(s-\tau) \mathrm{d} s \int_{t_{i-1}}^{t_{i}} \mu_{s} \mathrm{~d} s\left(\int_{t_{i-1}}^{t_{i}} \sigma_{s}^{2} \mathrm{~d} s\right)^{1 / 2} U_{i},
$$

Since $E\left(A_{n} \mid\left\{\sigma_{t}\right\}\right)=0$, from (2.6)-(2.8), we find that

$$
E\left(\hat{\sigma}_{\tau, G M}^{2} \mid\left\{\sigma_{t}\right\}\right)-E\left(\tilde{\hat{\sigma}}_{\tau, G M}^{2} \mid\left\{\sigma_{t}\right\}\right)=O\left(n^{-1}\right) .
$$

Therefore, it is sufficient to show that

$$
E\left(\tilde{\hat{\sigma}}_{\tau, G M}^{2} \mid\left\{\sigma_{t}\right\}\right)-\sigma_{\tau}^{2}=O_{p}\left(\frac{1}{n^{\beta} h_{n}^{1+\beta}}+h_{n}^{m+\alpha}\right) .
$$

By (2.8), we have that

$$
E\left[\left(\Delta \tilde{X}_{t_{i}}\right)^{2} \mid\left\{\sigma_{t}\right\}\right]=E\left[\left(\int_{t_{i-1}}^{t_{i}} \sigma_{s} \mathrm{~d} W_{s}\right)^{2} \mid\left\{\sigma_{t}\right\}\right]=\int_{t_{i-1}}^{t_{i}} \sigma_{s}^{2} \mathrm{~d} s,
$$

and

$$
\begin{aligned}
E\left(\tilde{\hat{\sigma}}_{\tau, G M}^{2} \mid\left\{\sigma_{t}\right\}\right)-\sigma_{\tau}^{2} & =\frac{1}{\delta} \sum_{i=1}^{n} \int_{t_{i-1}}^{t_{i}} K_{h}(s-\tau) \mathrm{d} s E\left(\left(\Delta \tilde{X}_{t_{i}}\right)^{2} \mid\left\{\sigma_{t}\right\}\right)-\sigma_{\tau}^{2} \\
& =\frac{1}{\delta} \sum_{i=1}^{n} \int_{t_{i-1}}^{t_{i}} K_{h}(s-\tau) \mathrm{d} s \int_{t_{i-1}}^{t_{i}} \sigma_{v}^{2} \mathrm{~d} v-\sigma_{\tau}^{2} .
\end{aligned}
$$


By the mean value theorem of integrals, there exist $\tilde{t}_{i} \in\left[t_{i-1}, t_{i}\right](i=1,2, \cdots, n)$ such that

$$
E\left(\tilde{\hat{\sigma}}_{\tau, G M}^{2} \mid\left\{\sigma_{t}\right\}\right)-\sigma_{\tau}^{2}=\sum_{i=1}^{n} K_{h}\left(\tilde{t}_{i}-\tau\right) \int_{t_{i-1}}^{t_{i}} \sigma_{v}^{2} \mathrm{~d} v-\sigma_{\tau}^{2} .
$$

We know that $K(u)$ satisfies the $\beta$ th Lipschiztz condition and $\sigma_{t}^{2}$ is bounded in $[0, T]$. It follows that

$$
\begin{aligned}
& \left|\sum_{i=1}^{n} K_{h}\left(\tilde{t}_{i}-\tau\right) \int_{t_{i-1}}^{t_{i}} \sigma_{v}^{2} \mathrm{~d} v-\int_{0}^{T} K_{h}(v-\tau) \sigma_{v}^{2} \mathrm{~d} v\right| \\
& =\left|\sum_{i=1}^{n} \int_{t_{i-1}}^{t_{i}}\left\{K_{h}\left(\tilde{t}_{i}-\tau\right)-K_{h}(v-\tau)\right\} \sigma_{v}^{2} \mathrm{~d} v\right| \\
& \leq C \sum_{i=1}^{n} \int_{t_{i-1}}^{t_{i}} \frac{1}{h_{n}}\left|\frac{\tilde{t}_{i}-v}{h_{n}}\right|^{\beta} \sigma_{v}^{2} \mathrm{~d} v \leq \frac{C \delta^{\beta}}{h_{n}^{1+\beta}} \int_{0}^{T} \sigma_{v}^{2} \mathrm{~d} v \leq \frac{C \delta^{\beta}}{h_{n}^{1+\beta}},
\end{aligned}
$$

hence

$$
\begin{aligned}
E\left(\tilde{\hat{\sigma}}_{\tau, G M}^{2} \mid\left\{\sigma_{t}\right\}\right)-\sigma_{\tau}^{2} & =\int_{0}^{T} K_{h}(v-\tau) \sigma_{v}^{2} \mathrm{~d} v-\sigma_{\tau}^{2}+O\left(\frac{\delta^{\beta}}{h_{n}^{1+\beta}}\right) \\
& =\int_{-\frac{\tau}{h_{n}}}^{\frac{T-\tau}{h_{n}}} K(u) \sigma_{\tau+u h_{n}}^{2} \mathrm{~d} u-\sigma_{\tau}^{2}+O\left(\frac{\delta^{\beta}}{h_{n}^{1+\beta}}\right) \\
& =\int_{-\frac{\tau}{h_{n}}}^{\frac{T-\tau}{h_{n}}} K(u) \sigma_{\tau+u h_{n}}^{2} \mathrm{~d} u-\sigma_{\tau}^{2} \int_{-\frac{\tau}{h_{n}}}^{\frac{T-\tau}{h_{n}}} K(u) \mathrm{d} u+O\left(\frac{\delta^{\beta}}{h_{n}^{1+\beta}}\right) .
\end{aligned}
$$

Recalling $f(t)=\sigma_{t}^{2}$ in the assumption (2) and $f(t)$ is $m$ times differentiable and its $m$ th derivative $f^{(m)}(t)$ satisfies the $\alpha$ th order Lipschitz condition in $L_{1}([0, T])$ where $m \geq 0$ and $\alpha>0$. By Taylor formula, we have that

$$
\begin{aligned}
& \int_{-\frac{\tau}{h_{n}}}^{\frac{T-\tau}{h_{n}}} K(u) \sigma_{\tau+u h_{n}}^{2} \mathrm{~d} u-\sigma_{\tau}^{2} \int_{-\frac{\tau}{h_{n}}}^{\frac{T-\tau}{h_{n}}} K(u) \mathrm{d} u \\
& =\sum_{j=1}^{m-1} \frac{f^{(j)}(\tau) h_{n}^{j}}{j !} \int_{-\frac{\tau}{h_{n}}}^{\frac{T-\tau}{h_{n}}} u^{j} K(u) \mathrm{d} u+\frac{h_{n}^{m}}{m !} \int_{-\frac{\tau}{h_{n}}}^{\frac{T-\tau}{h_{n}}} f^{(m)}\left(\tau+\theta u h_{n}\right) u^{m} K(u) \mathrm{d} u,
\end{aligned}
$$

where $|\theta| \leq 1$. Note that $\int_{-\infty}^{+\infty} u^{j} K(u) \mathrm{d} u=0, j=1,2, \cdots, m$ and $\int_{-\infty}^{\infty}|u|^{r}|K(u)| \mathrm{d} u<\infty$ where $r \geq m+\alpha$, we have

$$
\int_{-\frac{\tau}{h_{n}}}^{\frac{T-\tau}{h_{n}}} u^{j} K(u) \mathrm{d} u=-\int_{-\infty}^{-\frac{\tau}{h_{n}}} u^{j} K(u) \mathrm{d} u-\int_{\frac{T-\tau}{h_{n}}}^{\infty} u^{j} K(u) \mathrm{d} u=O\left(h_{n}^{r-j}\right),
$$

while

$$
\begin{aligned}
& \int_{-\frac{\tau}{h_{n}}}^{\frac{T-\tau}{h_{n}}} f^{(m)}\left(\tau+\theta u h_{n}\right) u^{m} K(u) \mathrm{d} u \\
& =\int_{-\frac{\tau}{h_{n}}}^{\frac{T-\tau}{h_{n}}}\left[f^{(m)}\left(\tau+\theta u h_{n}\right)-f^{(m)}(\tau)\right] u^{m} K(u) \mathrm{d} u \\
& -f^{(m)}(\tau) \int_{\infty}^{-\frac{\tau}{h_{n}}} u^{m} K(u) \mathrm{d} u-f^{(m)}(\tau) \int_{\frac{T-\tau}{h_{n}}}^{\infty} u^{m} K(u) \mathrm{d} u \\
& =\int_{-\frac{\tau}{h_{n}}}^{\frac{T-\tau}{h_{n}}}\left[f^{(m)}\left(\tau+\theta u h_{n}\right)-f^{(m)}(\tau)\right] u^{m} K(u) \mathrm{d} u+O\left(h_{n}^{r-m}\right),
\end{aligned}
$$


and

$$
\begin{aligned}
& E\left|\int_{-\frac{\tau}{h_{n}}}^{\frac{T-\tau}{h_{n}}}\left[f^{(m)}\left(\tau+\theta u h_{n}\right)-f^{(m)}(\tau)\right] u^{m} K(u) \mathrm{d} u\right| \\
& \leq \int_{-\frac{\tau}{h_{n}}}^{\frac{T-\tau}{h_{n}}} E\left|f^{(m)}\left(\tau+\theta u h_{n}\right)-f^{(m)}(\tau)\right||u|^{m}|K(u)| \mathrm{d} u \\
& \leq C \int_{-\frac{\tau}{h_{n}}}^{\frac{T-\tau}{h_{n}}}\left|\theta u h_{n}\right|^{\alpha}|u|^{m}|K(u)| \mathrm{d} u=O\left(h_{n}^{\alpha}\right) .
\end{aligned}
$$

Combining (2.17)-(2.20), we find that

$$
\int_{-\frac{\tau}{h_{n}}}^{\frac{T-\tau}{h_{n}}} K(u) \sigma_{\tau+u h_{n}}^{2} \mathrm{~d} u-\sigma_{\tau}^{2} \int_{-\frac{\tau}{h_{n}}}^{\frac{T-\tau}{h_{n}}} K(u) \mathrm{d} u=O_{p}\left(h_{n}^{m+\alpha}\right) .
$$

We obtain the desire result (2.11) from (2.16) and (2.21). Complete the proof.

Theorem 2.2. Assume that assumptions (1)-(3) hold, and $\beta>1 / 2$ in (b) of (3). If the bandwidth $h_{n}$ satisfies

$$
\begin{gathered}
n h_{n}^{(1 / 2+\beta) /(\beta-1 / 2)} \rightarrow \infty, \\
n h_{n}^{2(m+\alpha)+1} \rightarrow 0,
\end{gathered}
$$

then

$$
\frac{\sqrt{\delta^{-1} h_{n}}\left(\hat{\sigma}_{\tau, G M}^{2}-\sigma_{\tau}^{2}\right)}{\sqrt{2 \sigma_{\tau}^{4} \int_{-\infty}^{\infty} K^{2}(z) \mathrm{d} z}} \stackrel{d}{\longrightarrow} N(0,1) .
$$

Here we give some explanations for the conditions.

Remark 2.1. Obviously, $h_{n} \rightarrow 0$ from (2.23). Note that $(1 / 2+\beta) /(\beta-1 / 2)>1+1 / \beta$ as $\beta>1 / 2$, so that $n h_{n}^{1+1 / \beta} \rightarrow \infty$ from (2.23). It implies that the assumption (4) naturally holds under (2.22) and (2.23). Therefore, the assumption (4) doesn't appear in the assumption of Theorem 2.2.

Remark 2.2. Normally, the used kernel function $K(u)$ is derivative bounded. Thus, $K(u)$ usually satisfies $\beta \geq 1$ order Lipschitz condition. In this case, $1<(1 / 2+\beta) /(\beta-1 / 2) \leq 3$. Thus it can be seen that (2.22) is a reasonable condition.

Remark 2.3. The condition (2.23) is used in Kristensen [32] (2010, Theorem 3 and Theorem 4). It requires that $\sigma_{t}^{2}$ has smoothness. If $m=\alpha=1$, then (2.23) is equivalent to $n h_{n}^{5} \rightarrow 0$, while If $m=2$ and $\alpha=1$ (refer to Bandi and Nguyen [34] (2003) and Bandi and Phillips [35] (2003)), then (2.23) is equivalent to $n h_{n}^{7} \rightarrow 0$.

To prove the theorem, we introduce the following lemmas.

Lemma 2.1. (Rosenthal [36], 1970) Let $\left\{\xi_{i}: i \geq 1\right\}$ be a sequence of independent random variables with $E\left(\xi_{i}\right)=0$ and $E\left(\left|\xi_{i}\right|^{p}\right)<\infty$ for some $p \geq 2$. Then there exists constant $C$ no depending on $n$ such that

$$
E\left|\sum_{i=1}^{n} \xi_{i}\right|^{p} \leq C\left\{\sum_{i=1}^{n} E\left|\xi_{i}\right|^{p}+\left(\sum_{i=1}^{n} E \xi_{i}^{2}\right)^{p / 2}\right\} .
$$


Lemma 2.2. Let the kernel $K(u)$ satisfies $\int_{-\infty}^{+\infty}|K(u)| \mathrm{d} u<\infty$, $\int_{-\infty}^{+\infty}|u|^{r}|K(u)| \mathrm{d} u<\infty \quad$ where $r>0$, and there exists constants $\beta>0$ and $C>0$ such that

$$
|K(u)-K(v)| \leq C|v-v|^{\beta} \text { for any } u, v \in R .
$$

If $h \rightarrow 0$ and $n h^{1+1 / \beta} \rightarrow \infty$, then for any given $\tau \in(0, T)$,

$$
\delta \sum_{i=1}^{n}\left|K_{h}\left(s_{i}-\tau\right)\right|=\int_{-\infty}^{\infty}|K(u)| \mathrm{d} u+O\left(h^{r}+\left(n h^{1+1 / \beta}\right)^{-1}\right) .
$$

where $t_{i-1} \leq s_{i} \leq t_{i}$.

Proof By the mean value theorem, there exist $\hat{t}_{i} \in\left[t_{i-1}, t_{i}\right]$ such that

$$
\sum_{i=1}^{n} \int_{t_{i-1}}^{t_{i}}\left|K_{h}(s-\tau)\right| \mathrm{d} s=\delta_{n} \sum_{i=1}^{n}\left|K_{h}\left(\hat{t}_{i}-\tau\right)\right| .
$$

Hence,

$$
\begin{aligned}
\delta \sum_{i=1}^{n}\left|K_{h}\left(s_{i}-\tau\right)\right| & =\delta \sum_{i=1}^{n}\left|K_{h}\left(s_{i}-\tau\right)\right|-\delta \sum_{i=1}^{n}\left|K_{h}\left(\hat{t}_{i}-\tau\right)\right|+\sum_{i=1}^{n} \int_{t_{i-1}}^{t_{i}}\left|K_{h}(s-\tau)\right| \mathrm{d} s \\
& \leq \delta \sum_{i=1}^{n}|| K_{h}\left(s_{i}-\tau\right)|-| K_{h}\left(\hat{t}_{i}-\tau\right)\left|+\int_{0}^{T}\right| K_{h}(s-\tau) \mid \mathrm{d} s \\
& \leq \delta \sum_{i=1}^{n}\left|K_{h}\left(s_{i}-\tau\right)-K_{h}\left(\hat{t}_{i}-\tau\right)\right|+\int_{-\infty}^{\infty}|K(u)| \mathrm{d} u+O\left(h^{r}\right) \\
& =\int_{-\infty}^{\infty}|K(u)| \mathrm{d} u+O\left(h^{r}+\left(n h^{1+1 / \beta}\right)^{-\beta}\right) .
\end{aligned}
$$

Complete the proof.

\section{Proof of Theorem 2.2. Let}

$$
a_{i}(\mu, \sigma)=\int_{t_{i-1}}^{t_{i}} K_{h}(s-\tau) \mathrm{d} s \int_{t_{i-1}}^{t_{i}} \mu_{s} \mathrm{~d} s\left(\int_{t_{i-1}}^{t_{i}} \sigma_{s}^{2} \mathrm{~d} s\right)^{1 / 2} .
$$

Recalling $A_{n}$ defined in (2.9), we have that $A_{n}=\frac{2}{\delta} \sum_{i=1}^{n} a_{i}(\mu, \sigma) U_{i}$. Hence, by Lemma 2.1,

$$
\begin{aligned}
E\left(A_{n}^{4} \mid\left\{\mu_{t}, \sigma_{t}\right\}\right) & \leq \frac{C}{\delta^{4}}\left\{\sum_{i=1}^{n} a_{i}^{4}(\mu, \sigma)+\left(\sum_{i=1}^{n} a_{i}^{2}(\mu, \sigma)\right)^{2}\right\} \\
& \leq \frac{C}{\delta^{4}}\left\{\frac{\delta^{9}}{h_{n}^{3}} \sum_{i=1}^{n} \int_{t_{i-1}}^{t_{i}}\left|K_{h}(s-\tau)\right| \mathrm{d} s+\left(\frac{\delta^{4}}{h_{n}} \sum_{i=1}^{n} \int_{t_{i-1}}^{t_{i}}\left|K_{h}(s-\tau)\right| \mathrm{d} s\right)^{2}\right\}( \\
& \leq \frac{C \delta^{4}}{h_{n}^{2}} \leq \frac{C}{n^{4} h_{n}^{2}} .
\end{aligned}
$$

It implies that $E\left(A_{n}^{4}\right) \leq C n^{-4} h_{n}^{-2}$. From this and (2.6)-(2.9), it follows that, for any given $\varepsilon>0$

$$
\begin{aligned}
& P\left(\sqrt{\delta^{-1} h_{n}}\left|\hat{\sigma}_{\tau, G M}^{2}-\tilde{\hat{\sigma}}_{\tau, G M}^{2}\right|>\varepsilon\right) \\
& \leq P\left(\sqrt{\delta^{-1} h_{n}}\left|A_{n}\right|>\varepsilon / 2\right) \\
& \leq C\left(\delta^{-1} h_{n}\right)^{2} E\left(A_{n}^{4}\right) \\
& \leq C n^{-2} \rightarrow 0,
\end{aligned}
$$

that is 


$$
\sqrt{\delta^{-1} h_{n}}\left(\hat{\sigma}_{\tau, G M}^{2}-\tilde{\hat{\sigma}}_{\tau, G M}^{2}\right)=o_{p}(1) .
$$

Therefore, the remaining task is to show that

$$
\frac{\sqrt{\delta^{-1} h_{n}}\left(\tilde{\hat{\sigma}}_{\tau, G M}^{2}-\sigma_{\tau}^{2}\right)}{\sqrt{2 \sigma_{\tau}^{4} \int_{-\infty}^{\infty} K^{2}(z) \mathrm{d} z}} \stackrel{d}{\longrightarrow} N(0,1) .
$$

Let

$$
y_{n, i}(\tau)=\frac{1}{\delta} \int_{t_{i-1}}^{t_{i}} \sigma_{v}^{2} \mathrm{~d} v \int_{t_{i-1}}^{t_{i}} K_{h}(s-\tau) \mathrm{d} s,
$$

then

$$
\begin{gathered}
E\left(\tilde{\hat{\sigma}}_{\tau, G M}^{2} \mid\left\{\sigma_{t}\right\}\right)=\frac{1}{\delta} \sum_{i=1}^{n} \int_{t_{i-1}}^{t_{i}} \sigma_{v}^{2} \mathrm{~d} v \int_{t_{i-1}}^{t_{i}} K_{h}(s-\tau) \mathrm{d} s=\sum_{i=1}^{n} y_{n, i}(\tau) \\
\operatorname{Var}\left(\tilde{\hat{\sigma}}_{\tau, G M}^{2} \mid\left\{\sigma_{t}\right\}\right)=\frac{2}{\delta^{2}} \sum_{i=1}^{n}\left(\int_{t_{i-1}}^{t_{i}} \sigma_{v}^{2} \mathrm{~d} v\right)^{2}\left(\int_{t_{i-1}}^{t_{i}} K_{h}(s-\tau) \mathrm{d} s\right)^{2}=2 \sum_{i=1}^{n} y_{n, i}^{2}(\tau),
\end{gathered}
$$

while

$$
\begin{aligned}
& \sqrt{\delta^{-1} h_{n}} \frac{\tilde{\hat{\sigma}}_{\tau, G M}^{2}-\sigma_{\tau}^{2}}{\sqrt{2 \sigma_{\tau}^{4} \int_{-\infty}^{\infty} K^{2}(z) \mathrm{d} z}} \\
& =\frac{\tilde{\hat{\sigma}}_{\tau, G M}^{2}-\sum_{i=1}^{n} y_{n, i}(\tau)}{\sqrt{2 \sum_{i=1}^{n} y_{n, i}^{2}(\tau)}} \sqrt{\frac{\delta^{-1} h_{n} \sum_{i=1}^{n} y_{n, i}^{2}(\tau)}{\sigma_{\tau}^{4} \int_{-\infty}^{\infty} K^{2}(z) \mathrm{d} z}}+\sqrt{\delta^{-1} h_{n}} \frac{\sum_{i=1}^{n} y_{n, i}(\tau)-\sigma_{\tau}^{2}}{\sqrt{2 \sigma_{\tau}^{4} \int_{-\infty}^{\infty} K^{2}(z) \mathrm{d} z}} \\
& =: I_{1, n}(\tau) \cdot I_{2, n}(\tau)+I_{3, n}(\tau) \text {. }
\end{aligned}
$$

First, consider $I_{2, n}$. Denote that

$$
\begin{gathered}
J_{1, n}(\tau)=\frac{h_{n}}{\delta^{3}} \sum_{i=1}^{n}\left(\int_{t_{i-1}}^{t_{i}} \sigma_{v}^{2} \mathrm{~d} v\right)^{2}\left(\int_{t_{i-1}}^{t_{i}} K_{h}(s-\tau) \mathrm{d} s\right)^{2}-h_{n} \int_{0}^{T} \sigma_{s}^{4} K_{h}^{2}(s-\tau) \mathrm{d} s, \\
J_{2, n}(\tau)=h_{n} \int_{0}^{T} \sigma_{s}^{4} K_{h}^{2}(s-\tau) \mathrm{d} s-\sigma_{\tau}^{4} h_{n} \int_{0}^{T} K_{h}^{2}(s-\tau) \mathrm{d} s, \\
J_{3, n}(\tau)=\sigma_{\tau}^{4} h_{n} \int_{T}^{\infty} K_{h}^{2}(s-\tau) \mathrm{d} s+\sigma_{\tau}^{4} h_{n} \int_{-\infty}^{0} K_{h}^{2}(s-\tau) \mathrm{d} s .
\end{gathered}
$$

Let $z=(s-\tau) / h_{n}$, we have

$$
\begin{aligned}
& \delta^{-1} h_{n} \sum_{i=1}^{n} y_{n, i}^{2}(\tau)-\sigma_{\tau}^{4} \int_{-\infty}^{\infty} K^{2}(z) \mathrm{d} z \\
& =\frac{h_{n}}{\delta^{3}} \sum_{i=1}^{n}\left(\int_{t_{i-1}}^{t_{i}} \sigma_{v}^{2} \mathrm{~d} v\right)^{2}\left(\int_{t_{i-1}}^{t_{i}} K_{h}(s-\tau) \mathrm{d} s\right)^{2}-\sigma_{\tau}^{4} h_{n} \int_{-\infty}^{\infty} K_{h}^{2}(s-\tau) \mathrm{d} s \\
& =J_{1, n}(\tau)+J_{2, n}(\tau)+J_{3, n}(\tau) .
\end{aligned}
$$

By the mean value theorem, there exists $v_{i}, s_{i}, \tilde{s}_{i} \in\left[t_{i-1}, t_{i}\right](i=1,2, \cdots, n)$, such that

$$
\begin{aligned}
J_{1, n}(\tau) & =\frac{h_{n}}{\delta^{3}} \sum_{i=1}^{n}\left(\int_{t_{i-1}}^{t_{i}} \sigma_{v}^{2} \mathrm{~d} v\right)^{2}\left(\int_{t_{i-1}}^{t_{i}} K_{h}(s-\tau) \mathrm{d} s\right)^{2}-h_{n} \sum_{i=1}^{n} \int_{t_{i-1}}^{t_{i}} \sigma_{s}^{4} K_{h}^{2}(s-\tau) \mathrm{d} s \\
& =\delta h_{n} \sum_{i=1}^{n} \sigma_{v_{i}}^{4} K_{h}^{2}\left(s_{i}-\tau\right)-\delta h_{n} \sum_{i=1}^{n} \sigma_{\tilde{s}_{i}}^{4} K_{h}^{2}\left(\tilde{s}_{i}-\tau\right) \\
& =\delta h_{n} \sum_{i=1}^{n}\left(\sigma_{v_{i}}^{4}-\sigma_{\tilde{s}_{i}}^{4}\right) K_{h}^{2}\left(s_{i}-\tau\right)+\delta h_{n} \sum_{i=1}^{n} \sigma_{\tilde{s}_{i}}^{4}\left\{K_{h}^{2}\left(s_{i}-\tau\right)-K_{h}^{2}\left(\tilde{s}_{i}-\tau\right)\right\} \\
& =: Q_{1, n}(\tau)+Q_{2, n}(\tau) .
\end{aligned}
$$


Using Lemma 2.2, we get that

$$
\begin{aligned}
E\left|Q_{1, n}(\tau)\right| & \leq C \delta h_{n} \sum_{i=1}^{n} E\left|\sigma_{v_{i}}^{2}-\sigma_{\tilde{s}_{i}}^{2}\right| K_{h}^{2}\left(s_{i}-\tau\right) \\
& \leq C \delta^{\lambda} \sum_{i=1}^{n} \delta\left|K_{h}\left(s_{i}-\tau\right)\right| \leq C \delta^{\lambda},
\end{aligned}
$$

and

$$
\begin{aligned}
\left|Q_{2, n}(\tau)\right| & \leq C \delta h_{n} \sum_{i=1}^{n}\left|K_{h}\left(s_{i}-\tau\right)-K_{h}\left(\tilde{s}_{i}-\tau\right)\right| \cdot\left|K_{h}\left(s_{i}-\tau\right)+K_{h}\left(\tilde{s}_{i}-\tau\right)\right| \\
& \leq \frac{C \delta^{1+\beta}}{h_{n}^{\beta}} \sum_{i=1}^{n}\left|K_{h}\left(s_{i}-\tau\right)+K_{h}\left(\tilde{s}_{i}-\tau\right)\right| \leq C\left(\frac{\delta}{h_{n}}\right)^{\beta}
\end{aligned}
$$

thus

$$
J_{1, n}(\tau)=O_{p}\left(n^{-\lambda}+\left(n h_{n}\right)^{-\beta}\right) .
$$

Using the integral transform $u=(s-\tau) / h_{n}$ for $J_{2, n}(\tau)$, and by (2) and (3), we known that

$$
\begin{aligned}
& E\left|J_{2, n}(\tau)\right|=E\left|\int_{-\tau / h_{n}}^{(T-\tau) / h_{n}}\left(\sigma_{u h_{n}+\tau}^{4}-\sigma_{\tau}^{4}\right) K^{2}(u) \mathrm{d} u\right| \\
& \leq C \int_{-\tau / h_{n}}^{(T-\tau) / h_{n}} E\left|\sigma_{u h_{n}+\tau}^{2}-\sigma_{\tau}^{2}\right| K^{2}(u) \mathrm{d} u \leq C h_{n}^{\lambda} \int_{-\infty}^{\infty}|u|^{\lambda}|K(u)| \mathrm{d} u \leq C h_{n}^{\lambda} .
\end{aligned}
$$

Note that $\int_{-\infty}^{+\infty}|u|^{\alpha}|K(u)| \mathrm{d} u<\infty$, we find that

$$
\begin{aligned}
& \left|J_{3, n}(\tau)\right| \leq C \sigma_{\tau}^{4} \int_{(T-\tau) / h_{n}}^{\infty}|K(u)| \mathrm{d} u+C \sigma_{\tau}^{4} \int_{-\infty}^{-\tau / h_{n}}|K(u)| \mathrm{d} u \\
& \leq \frac{C \sigma_{\tau}^{4} h_{n}^{\alpha}}{(T-\tau)^{\alpha}} \int_{(T-\tau) / h_{n}}^{\infty}|u|^{\alpha}|K(u)| \mathrm{d} u+\frac{C \sigma_{\tau}^{4} h_{n}^{\alpha}}{-\tau^{\alpha}} \int_{-\infty}^{-\tau / h_{n}}|u|^{\alpha}|K(u)| \mathrm{d} u \leq C h_{n}^{\alpha} .
\end{aligned}
$$

Combining (2.42) and (2.46)-(2.48), we have

$$
\delta^{-1} h_{n} \sum_{i=1}^{n} y_{n, i}^{2}(\tau)-\sigma_{\tau}^{4} \int_{-\infty}^{\infty} K^{2}(z) \mathrm{d} z=o_{p}(1) .
$$

It follows that

$$
I_{2, n}(\tau) \stackrel{p}{\longrightarrow} 1 .
$$

Next, consider $I_{1, n}$. We defined $c_{n, i}(\tau)=y_{n, i}(\tau) / \sqrt{\sum_{i=1}^{n} y_{n, i}^{2}(\tau)}$, thus

$$
I_{1, n}(\tau) \stackrel{\text { law }}{=} \sum_{i=1}^{n} c_{n, i}(\tau) \bar{U}_{i}^{2},
$$

where $\bar{U}_{i}^{2}=\left(U_{i}^{2}-1\right) / \sqrt{2}$ are independent and identically distributed random variables with mean zero and variance one. According to (2.49), we obtain

$$
\begin{aligned}
c_{n, i}(\tau) & =\frac{y_{n, i}(\tau)}{\sqrt{\frac{T}{n h_{n}} \sigma_{\tau}^{4} \int_{-\infty}^{\infty} K^{2}(z) \mathrm{d} z+o_{p}\left(\frac{1}{n h_{n}}\right)}}=\frac{\frac{1}{\delta h_{n}} \int_{t_{i-1}}^{t_{i}} \sigma_{v}^{2} \mathrm{~d} v \int_{t_{i-1}}^{t_{i}} K\left(\frac{s-\tau}{h_{n}}\right) \mathrm{d} s}{\sqrt{\frac{T}{n h_{n}} \sigma_{\tau}^{4} \int_{-\infty}^{\infty} K^{2}(z) \mathrm{d} z+o_{p}\left(\frac{1}{n h_{n}}\right)}} \\
& \leq \frac{C}{\sqrt{\frac{T}{n h_{n}} \sigma_{\tau}^{4} \int_{-\infty}^{\infty} K^{2}(z) \mathrm{d} z+o_{p}\left(\frac{1}{n h_{n}}\right)}} \leq C\left(\frac{1}{n h_{n}}\right)^{1 / 2},
\end{aligned}
$$


and $\sum_{i=1}^{n} c_{n, i}^{2}(\tau)=1$, by Barndorff-Nielsen and Shephard [14] (2006, Cor. 3.1), we obtain

$$
I_{1, n}(\tau) \mid\left\{\sigma_{t}\right\} \stackrel{d}{\longrightarrow} N(0,1) .
$$

Finally, consider $I_{3, n}$. By (2.3), we known

$$
\sum_{i=1}^{n} y_{n, i}(\tau)-\sigma_{\tau}^{2}=O_{p}\left(n^{-1}+\frac{1}{n^{\beta} h_{n}^{1+\beta}}+h_{n}^{m+\alpha}\right),
$$

then

$$
\begin{aligned}
I_{3, n}(\tau) & =O_{p}\left(n^{-1}+\frac{1}{n^{\beta} h_{n}^{1+\beta}}+h_{n}^{m+\alpha}\right)\left(n h_{n}\right)^{1 / 2} \\
& =O_{p}\left(n^{-1 / 2} h_{n}^{1 / 2}+\frac{1}{n^{\beta-1 / 2} h_{n}^{1 / 2+\beta}}+n^{1 / 2} h_{n}^{m+\alpha+1 / 2}\right) \\
& =O_{p}\left(n^{-1 / 2} h_{n}^{1 / 2}+\frac{1}{\left(n h_{n}^{(1 / 2+\beta) /(\beta-1 / 2)}\right)^{\beta-1 / 2}}+\left(n h_{n}^{2 m+2 \alpha+1}\right)^{1 / 2}\right) .
\end{aligned}
$$

By (2.22) and (2.23), we obtain

$$
I_{3, n}(\tau)=o_{p}(1) .
$$

Therefore, it follows (2.34) from (2.50), (2.53) and (2.56). Complete the proof.

\section{Simulation}

Here, we will show the performance of the Gasser-Müller integrated type spot volatility estimator $\hat{\sigma}_{\tau, G M}^{2}$ (see 1.5) proposed in this paper and compare it with the kernel estimator $\hat{\sigma}_{\tau}^{2}$ proposed by Kristensen [32] (2010). We consider the following stochastic volatility model as referred in Kanaya and Kristensen [37] (2016),

$$
\begin{gathered}
\mathrm{d} X_{t}=\sigma_{t} \mathrm{~d} W_{1, t}, \\
\mathrm{~d} \sigma_{t}^{2}=\beta\left(\alpha-\sigma_{t}^{2}\right) \mathrm{d} t+\kappa \sigma_{t}^{2} \mathrm{~d} W_{2, t},
\end{gathered}
$$

where $W_{1, t}$ and $W_{2, t}$ are independent standard Brownian motions, parameters are respectively $\beta=0.510, \alpha=0.476$ and $\kappa^{2}=0.0518$ (refer to Kanaya and Kristensen [37] (2016), page 888). In order to simulate the above model, we use Euler discretization scheme (see Kloeden and Platten [7], 1999) to obtain the following model.

$$
\begin{gathered}
\Delta X_{i \delta}=\sigma_{(i-1) \delta} \sqrt{\delta} \varepsilon_{1, i}, \\
\Delta \sigma_{i \delta}^{2}=\beta\left(\alpha-\sigma_{(i-1) \delta}^{2}\right) \delta+\kappa \sigma_{(i-1) \delta}^{2} \sqrt{\delta} \varepsilon_{2, i} .
\end{gathered}
$$

where $\left\{\varepsilon_{1, i}\right\}$ and $\left\{\varepsilon_{2, i}\right\}$ are i.i.d $N(0,1), \delta>0$ is the length of the discretization step.

In the actual operation of the simulation, we assume that the drift term $\mu_{t}=0$, and the observed time is $T=2$ (48 hours). The specific steps are as follows:

1) Get the true value $\left\{\sigma_{t}\right\}$. According to model (3.2), the truth sequence 
$\left\{\sigma_{t}\right\}$ of $\sigma_{t}$ is obtained, because the discretized model is approximated to the original continuity model and the simulation interval is as small as possible, so the time interval $\delta=\Delta / 100$ is selected in model (3.2), where $\Delta^{-1}=3 \times 60 \times 24 \times 2$.

2) Produce samples. $\Delta X_{i \delta}$ is sampled at 20 seconds, 1 minute, 5 minutes, 10 minutes, 15 minutes and 30 minutes sampling frequency. Model (3.1) is used to generate simulated sample $\left\{\Delta X_{i \delta}, i=1,2, \cdots, n\right\}$. The numbers of samples corresponding to various sampling frequencies are

$$
\delta^{-1}=3 \times 60 \times 24 \times 2,60 \times 24 \times 2,12 \times 24 \times 2,6 \times 24 \times 2,4 \times 24 \times 2,2 \times 24 \times 2,
$$

respectively. At the same time, the sample is repeated 400 times and 400 sets of sample data are obtained for each sampling case. Define

$\tilde{\sigma}_{t_{i}, j}^{2}, i=1,2, \cdots, n ; j=1,2, \cdots, 400$ as the estimated value at time $t_{i}$ using the group $j$ sample data.

3) Choose kernel function and bandwidth. In the process of calculating estimators $\hat{\sigma}_{\tau, G M}^{2}$ and $\hat{\sigma}_{\tau}$, we choose the Gaussian kernel. Since the choice of the kernel function has a great influence on estimates on the boundary (see Silverman [38] (1986)), in order to avoid the boundary effects, we only considered estimated values of the $[1 / 2,3 / 2]$. Because the selection of bandwidth has a great influence on the internal estimation performance, we apply the cross-validated mentioned in Kristensen [32] (2010) to obtain the optimal bandwidth, namely, the optimum bandwidth is the minimum bandwidth for

$$
\widehat{\operatorname{ISE}}\left(h_{n}\right)=\sum_{i=1}^{n} I\left\{T_{l} \leq t_{i-1} \leq T_{u}\right\}\left[\frac{\left(\Delta X_{t_{i}}\right)^{2}}{\delta}-\hat{\sigma}_{-i, t_{i}}^{2}\right],
$$

where $\hat{\sigma}_{-i, t_{i}}^{2}$ is based on the samples removed from the $i$ th sample to calculate the spot volatility estimator of the time $t_{i}=i \delta, T_{l}$ and $T_{u}$ are severally the upper and lower limits of the time span we consider, $T_{l}=1 / 2, T_{u}=3 / 2$. Using the simulated sample $\left\{\Delta X_{i \delta}\right\}$ and (3.3), we acquire the optimal bandwidth of the kernel spot volatility estimator and the GM type spot volatility estimator under the different time intervals.

We want to measure the accuracy of the estimator (i.e. estimation effect) by the bias, the variance and the integrated mean square error of this estimator. The calculation formulas for the bias, the variance and the integrated mean square error of the estimators are respectively

$$
\begin{gathered}
\operatorname{Bias}^{2}=\frac{1}{n} \sum_{i=1}^{n}\left(\frac{1}{400} \sum_{j=1}^{400} \tilde{\sigma}_{t_{i}, j}^{2}-\sigma_{t_{i}}^{2}\right)^{2}, \\
\operatorname{Var}=\frac{1}{n} \sum_{i=1}^{n} \frac{1}{400} \sum_{j=1}^{400}\left(\tilde{\sigma}_{t_{i}, j}^{2}-\frac{1}{400} \sum_{j=1}^{400} \tilde{\sigma}_{t_{i}, j}^{2}\right)^{2},
\end{gathered}
$$

and

$$
\operatorname{IMSE}=\frac{1}{n} \sum_{i=1}^{n} \frac{1}{400} \sum_{j=1}^{400}\left(\tilde{\sigma}_{t_{i}, j}^{2}-\sigma_{t_{i}}^{2}\right)^{2} .
$$

Obviously, $\quad$ IMSE $=\mathrm{Var}+\mathrm{Bias}^{2}$. 
Table 1. Performance in interior.

\begin{tabular}{ccccc}
\hline Sampling frequency & Type & Bias $^{2}$ & Var & IMSE \\
\hline \multirow{2}{*}{20 seconds } & $\hat{\sigma}_{\tau, G M}^{2}$ & 0.46374 & 7.65629 & 8.12003 \\
& $\hat{\sigma}_{\tau}$ & 0.46388 & 7.65646 & 8.12033 \\
1 minute & $\hat{\sigma}_{\tau, G M}^{2}$ & 1.59960 & 15.46301 & 17.06261 \\
& $\hat{\sigma}_{\tau}$ & 1.60059 & 15.46395 & 17.06454 \\
5 minutes & $\hat{\sigma}_{\tau, G M}^{2}$ & 2.75254 & 28.00331 & 30.75585 \\
& $\hat{\sigma}_{\tau}$ & 2.76279 & 28.00738 & 30.77016 \\
10 minutes & $\hat{\sigma}_{\tau, G M}^{2}$ & 4.16189 & 38.34402 & 42.50591 \\
& $\hat{\sigma}_{\tau}$ & 4.16406 & 38.38035 & 42.54441 \\
& $\hat{\sigma}_{\tau, G M}^{2}$ & 5.04384 & 47.84588 & 52.88972 \\
15 minutes & $\hat{\sigma}_{\tau}$ & 5.02933 & 47.90483 & 52.93416 \\
& $\hat{\sigma}_{\tau, G M}^{2}$ & 7.52807 & 89.87464 & 97.40271 \\
& $\hat{\sigma}_{\tau}$ & 7.33064 & 90.20958 & 97.54365 \\
\hline
\end{tabular}

[Note: Integrated sq.bias $\left(\times 10^{-4}\right)$, variance $\left(\times 10^{-4}\right)$, and IMSE $\left(\times 10^{-4}\right)$ of estimators].

The bias, the variance and the integrated mean square error of the GM instantaneous volatility estimator and the kernel instantaneous volatility estimator under the different time intervals are reported in Table 1.

According to the simulation results shown in Table 1, we find that the higher the sampling frequency, the better the accuracy of both the GM type spot volatility estimator $\hat{\sigma}_{\tau, G M}^{2}$ and the kernel spot volatility estimator $\hat{\sigma}_{\tau}$, indicating that both estimators are suitable for high frequency data. At the same time, we see that the IMSE of $\hat{\sigma}_{\tau, G M}^{2}$ is slightly smaller than that of $\hat{\sigma}_{\tau}$. This shows that the GM type spot volatility estimator provides a selection method for estimating the spot volatility, especially in high frequency data environment, its estimation performance is better.

\section{Conclusion}

We propose a Gasser-Müller type spot volatility estimator for diffusion process, and prove its asymptotic unbiasedness and asymptotic normality under mild conditions. By simulation analysis, we find that the GM type spot volatility estimator is a better estimator. Therefore, it provides a selection method for estimating the spot volatility in high frequency data environment.

\section{Funding}

This research was supported by the National Natural Science Foundation of China [grant number 11461009], the Innovation Project of Guangxi Graduate Education [grant number XYCSZ2017075] and the Scientific research project of the Guangxi Colleges and Universities [grant number KY2015YB345]. 


\section{Authors' Contributions}

All authors carried out the mathematical studies, and read and approved the final manuscript.

\section{Conflicts of Interest}

The authors declare that they have no competing interests.

\section{References}

[1] Barndorff-Nielsen, O.E. and Shephard, N. (2002) Econometric Analysis of Realized Volatility and Its Use in Estimating Stochastic Volatility Models. Journal of the Royal Statistical Society: Series B (Statistical Methodology), 64, 253-280. https://doi.org/10.1111/1467-9868.00336

[2] Christensen, K. and Podolski, M. (2005) Asymptotic Theory for Range-Based Estimation of Integrated Variance of a Continuous Semi-Martingale. Technical Reports.

[3] Li, C., Guo, E. and Bao, M. (2012) A Nonparametric Estimate of Integrated CrossVolatility. Acta Scientiarum Naturalium Universitatis Sunyatseni, 52, 55-58.

[4] Zhang, L., Mykland, P.A. and Aït-Sahalia, Y. (2005) A Tale of Two Time Scales: Determining Integrated Volatility with Noisy High-Frequency Data. Journal of the American Statistical Association, 100, 1394-1411. https://doi.org/10.1198/016214505000000169

[5] Hansen, P., Large, J.H. and Lunde, A. (2008) Moving Average-Based Estimators of Integrated Variance. Econometric Reviews, 27, 79-111. https://doi.org/10.1080/07474930701853640

[6] Li, C.X., Liang, X.L., Jing, B.Y. and Kong, X.B. (2013) The Asymptotics of the Integrated Self-Weighted Cross Volatility Estimator. Journal of Statistical Planning \& Inference, 143, 1708-1718. https://doi.org/10.1016/j.jspi.2013.05.003

[7] Jing, B., Liu, Z. and Kong, X. (2015) Estimating the Volatility Functionals with Multiple Transactions. Econometric Theory, 33, 331-365. https://doi.org/10.1017/S0266466615000420

[8] Li, Y., Xie, S. and Zheng, X. (2016) Efficient Estimation of Integrated Volatility Incorporating Trading Information. Journal of Econometrics, 195, 33-50. https://doi.org/10.1016/j.jeconom.2016.05.017

[9] Merton, R.C. (1976) Option Pricing When Underlying Stock Returns Are Discontinuous. Journal of Financial Economics, 3, 125-144. https://doi.org/10.1016/0304-405X(76)90022-2

[10] Duffie, D., Pan, J. and Singleton, K. (2000) Transform Analysis and Asset Pricing for Affine Jump-Diffusions. Econometrica, 68, 1343-1376. https://doi.org/10.1111/1468-0262.00164

[11] Pan, J. (2002) The Jump-Risk Premia Implicit in Options: Evidence from an Integrated Time Series Study. Journal of Financial Economics, 63, 3-50. https://doi.org/10.1016/S0304-405X(01)00088-5

[12] Eraker, B., Johannes, M. and Polson, N. (2003) The Impact of Jumps in Equity Index Volatility and Returns. Journal of Finance, 58, 1269-1300. https://doi.org/10.1111/1540-6261.00566

[13] Barndorff-Nielsen, O.E. and Shephard, N. (2004) Power and Bipower Variation with Stochastic Volatility and Jumps. Economics Papers, 2, 1-37. 
https://doi.org/10.1093/jifinec/nbh001

[14] Barndorff-Nielsen, O.E. and Shephard, N. (2006) Econometrics of Testing for Jumps in Financial Economics Using Bipower Variation. Journal of Financial Econometrics, 4, 1-30. https://doi.org/10.1093/jifinec/nbi022

[15] Mancini, C. (2004) Estimation of the Characteristics of the Jumps of a General Poisson-Diffusion Model. Scandinavian Actuarial Journal, 2004, 42-52. https://doi.org/10.1080/034612303100170091

[16] Mancini, C. (2009) Non-Parametric Threshold Estimation for Models with Stochastic Diffusion Coefficient and Jumps. Scandinavian Journal of Statistics, 36, 270-296. https://doi.org/10.1111/j.1467-9469.2008.00622.x

[17] Christensen, K. and Podolskij, M. (2012) Asymptotic Theory of Range-Based Multipower Variation. Journal of Financial Econometrics, 10, 417-456.

https://doi.org/10.1093/jifinec/nbr019

[18] Todorov, V. (2009) Estimation of Continuous-Time Stochastic Volatility Models with Jumps Using High-Frequency Data. Journal of Econometrics, 148, 131-148. https://doi.org/10.1016/j.jeconom.2008.10.005

[19] Todorov, V. (2010) Variance Risk-Premium Dynamics: The Role of Jumps. Review of Financial Studies, 23, 345-383. https://doi.org/10.1093/rfs/hhp035

[20] At-Sahalia, Y. and Jacod, J. (2009) Estimating the Degree of Activity of Jumps in High Frequency Data. Annals of Statistics, 37, 2202-2244. https://doi.org/10.1214/08-AOS640

[21] At-Sahalia, Y. and Jacod, J. (2011) Analyzing the Spectrum of Asset Returns: Jump and Volatility Components in High Frequency Data. Journal of Economic Literature, 50, 1007-1050. https://doi.org/10.1257/jel.50.4.1007

[22] Tauchen, G. and Zhou, H. (2011) Realized Jumps on Financial Markets and Predicting Credit Spreads. Journal of Econometrics, 160, 102-118. https://doi.org/10.1016/j.jeconom.2010.03.023

[23] Foster, D.P. and Nelson, D.B. (1996) Continuous Record Asymptotics for Rolling Sample Variance Estimators. Modelling Stock Market Volatility, 64, 291-329. https://doi.org/10.1016/B978-012598275-7.50011-9

[24] Andreou, E. and Ghysels, E. (2002) Rolling-Sample Volatility Estimators: Some New Theoretical, Simulation and Empirical Results. Journal of Business \& Economic Statistics, 20, 363-376. https://doi.org/10.1198/073500102288618504

[25] Fan, J. and Wang, Y. (2008) Spot Volatility Estimation for High-Frequency Data. Statistics and Its Interface, 1, 279-288. https://doi.org/10.4310/SII.2008.v1.n2.a5

[26] Shigeyoshi, O. and Simona, S. (2011) An Improved Two-Step Regularization Scheme for Spot Volatility Estimation. Economic Notes, 40, 107-134.

https://doi.org/10.1111/j.1468-0300.2011.00233.x

[27] Zu, Y. and Boswijk, H.P. (2014) Estimating Spot Volatility with High-Frequency Financial Data. Journal of Econometrics, 181, 117-135. https://doi.org/10.1016/j.jeconom.2014.04.001

[28] Yu, C., Fang, Y., Li, Z., Zhang, B. and Zhao, X. (2014) Non Parametric Estimation of High-Frequency Spot Volatility for Brownian Semimartingale with Jumps. Journal of Time Series Analysis, 35, 572-591. https://doi.org/10.1111/jtsa.12082

[29] Mancini, C., Mattiussi, V. and Renò, R. (2015) Spot Volatility Estimation Using Delta Sequences. Finance and Stochastics, 19, 261-293. https://doi.org/10.1007/s00780-015-0255-1

[30] Curato, I.V., Mancino, M.E. and Recchioni, M.C. (2018) Spot Volatility Estimation 
Using the Laplace Transform. Econometrics and Statistics, 6, 22-43.

https://doi.org/10.1016/j.ecosta.2016.07.002

[31] Liu, Q., Liu, Y., Liu, Z. and Wang, L. (2018) Estimation of Spot Volatility with Superposed Noisy Data. The North American Journal of Economics and Finance, 44, 62-79. https://doi.org/10.1016/j.najef.2017.11.004

[32] Kristensen, D. (2010) Nonparametric Filtering of the Realized Spot Volatility: A Kernel-Based Approach. Econometric Theory, 26, 60-93. https://doi.org/10.1017/S0266466609090616

[33] Gasser, T. and Müller, H.G. (1979) Kernel Estimation of Regression Functions. In: Gasser, T. and Rosenblatt, M., Eds., Smoothing Techniques for Curve Estimation, Springer-Verlag, Berlin, 23-68. https://doi.org/10.1007/BFb0098489

[34] Bandi, F.M. and Nguyen, T.H. (2003) On the Functional Estimation of Jump-Diffusion Models. Journal of Econometrics, 116, 293-328. https://doi.org/10.1016/S0304-4076(03)00110-6

[35] Bandi, F.M. and Phillips, P.C.B. (2003) Fully Nonparametric Estimation of Scalar Diffusion Models. Econometrica, 71, 241-283. https://doi.org/10.1111/1468-0262.00395

[36] Rosenthal, H.P. (1970) On the Estimations of Sums of Independent Random Variables. Israel Journal of Mathematics, 8, 273-303. https://doi.org/10.1007/BF02771562

[37] Kanaya, S. and Kristensen, D. (2016) Estimation of Stochastic Volatility Models by Nonparametric Filtering. Econometric Theory, 32, 861-916. https://doi.org/10.1017/S0266466615000079

[38] Silverman, B.W. (1986) Density Estimation for Statistics and Data Analysis. Chapman and Hall, London. https://doi.org/10.1007/978-1-4899-3324-9 